\title{
How soaring agricultural prices will impact the way we do feed business
}

\author{
Supachai Pathumnakul ${ }^{1 *}$, Kullapapruk Piewthongngam ${ }^{2}$ \\ ${ }^{1}$ Supply chain and logistics research unit, Department of Industrial Engineering, Faculty of Engineering, Khon Kaen University, Khon Kaen \\ 40002, Thailand. \\ ${ }^{2}$ E-saan Center for Business and Economic Research, Faculty of Management Science, Khon Kaen University, Khon Kaen 40002, Thailand.
}

\begin{abstract}
The rising price of agricultural products leads to frequent change of feed recipe, which can cause a high number of reprocessing batches, elevating the overall cost of production. In this study, we proposed an artificial neural network to predict production rate. The conversion of production rate to production cost, the tips for data collection as well as tips for implementation of new feed cost estimation are also discussed. Being able to estimate production rate enables feed mills to improve their operations. In this study, we elaborate its application to feed scheduling (although the applications can be extended to other aspects such as productivity improvement, which goes beyond the scope of this particular study).
\end{abstract}

Key Words: artificial neural network, feed cost estimation, feed scheduling

\section{Impacto do aumento dos preços agrícolas sobre os negócios do ramo alimentício}

RESUMO - O aumento do preço dos produtos agrícolas leva à mudança frequente de receita para alimentação animal, que pode resultar em elevado número de reprocessamento de lotes, aumentando o custo total de produção. Neste estudo, propusemos uma rede neural artificial para prever a taxa de produção. A conversão da taxa de produção para custo de produção, as dicas para a coleta de dados, bem como as dicas para a aplicação de nova estimativa de custo de alimentação, também são discutidas. A capacidade de estimar a taxa de produção e habilitar as fábricas de ração a melhorarem suas operações. Neste estudo, elaboramos a sua aplicação para abastecer a programação (embora os aplicativos podem ser estendidos a outros aspectos, como a melhoria da produtividade, o que ultrapassa o âmbito deste estudo).

Palavras-chave: abastecimento de programação, estimativa de custos com alimentação, rede neural artificial

\section{Introduction}

The rising use of biomass for energy production puts the food and feed industry in direct competition with car drivers for the same agricultural output. As ethanol demand rises, prices of agricultural products are now highly correlated with gasoline prices. As shown in Figure 1, the price of raw materials such as yellow corn, rice bran, soybean meal and cassava has fluctuated highly since 2006. The situation will continue as long as the same agricultural output is used for both the food and automobile industries. The recent fluctuation in terms of the cost of feed ingredient will prove to be a challenge to feed manufacturing practices across the globe in many ways. Firstly, the rising raw material prices will also trigger feed prices to rise. This is because raw material costs usual make up for more than $70 \%$ of the total cost of feed. Secondly, feed mill garners more interest to control its raw material supply. Integration with their suppliers is expected to be on the rise as well. Thirdly, price turbulence leads to frequent change of feed recipes. For example (Table 1), in September 2009, the percent inclusion of cassava chips in the swine feed of a Thai feed mill was 12.76, while it was not included in the recipe in October 2009. Hence, the mill has to deal with frequently recipe change in this present era of turbulent prices. The first and the second effects are strategically manageable. However, for the third effect, feed mill needs to alter their business process in order to remain efficienct. In this study, we focus on how the feed mill should change its cost estimation and feed scheduling in order to cope with frequent recipe changes.

\section{The cost estimation}

Since the 1960s, feed recipe has been obtained using linear programming. The least-cost formulation determines the cheapest blend of available raw materials that meet certain nutritional requirements. Even though the nutritional requirements are met in the calculations, the minimum cost considered is raw material cost while the production cost is

Corresponding author: supa_pat@kku.ac.th 

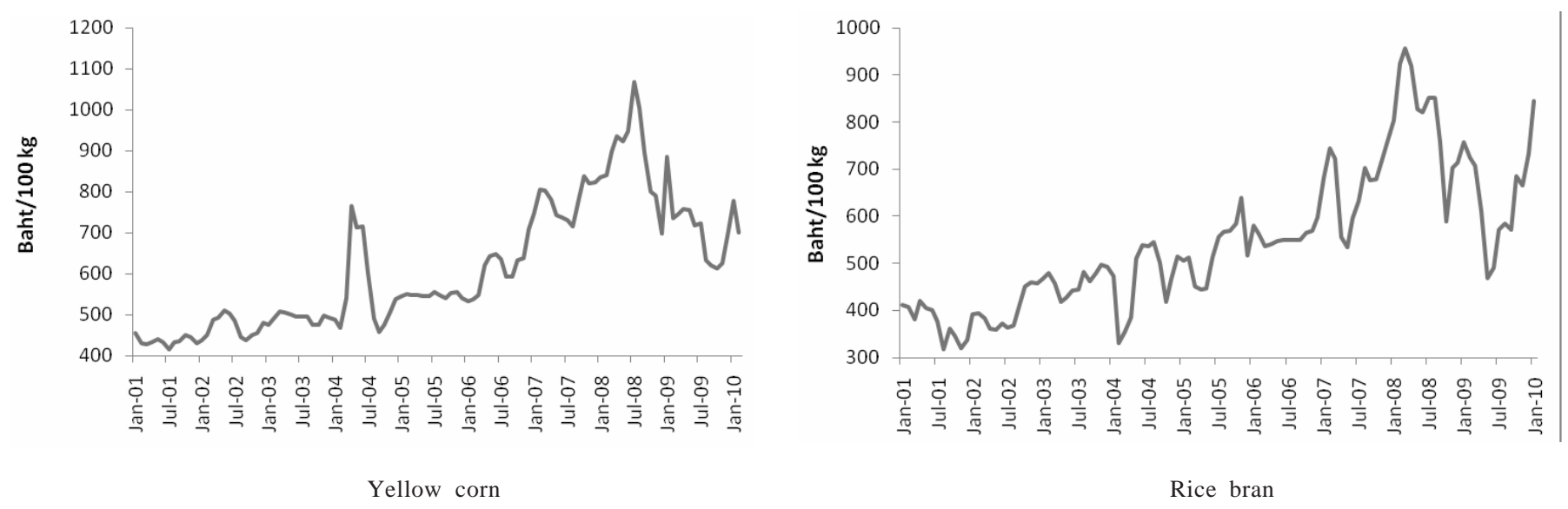

Rice bran
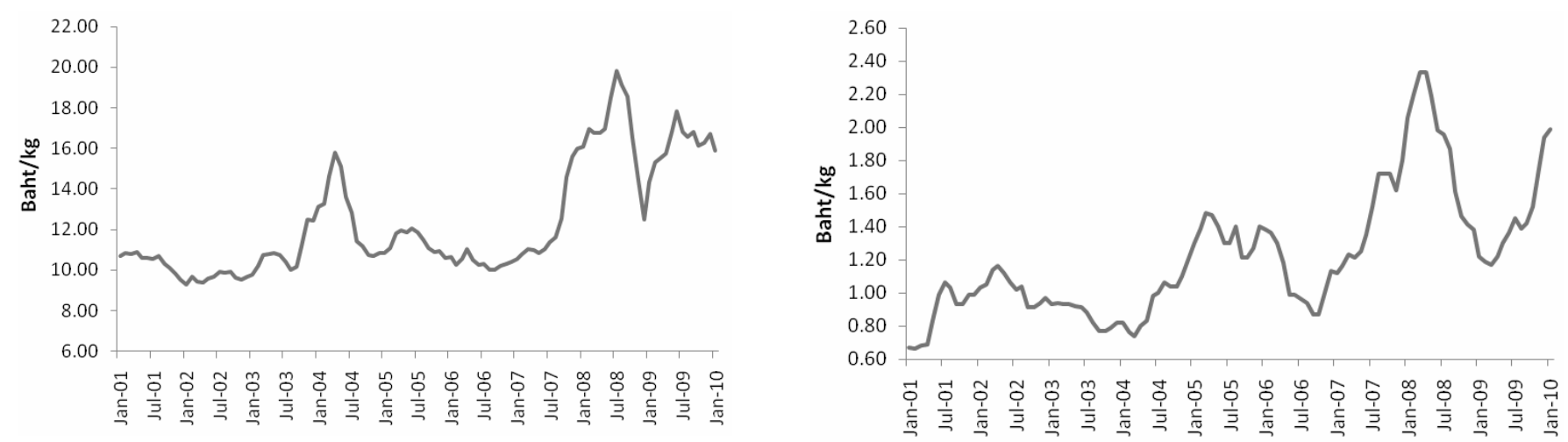

Soybean meal

Cassava

Figure 1 - Price of main raw materials used for feed products.

Table 1 - A swine feed formula suggested by least cost formulation in September and October 2009

\begin{tabular}{lcc}
\hline Month & Raw material type & \% inclusion \\
\hline September & Yellow corn & 22.45 \\
& Cassava chip & 12.74 \\
& Rice solvent bran & 17.02 \\
Rice bran dry season & 18.35 \\
& Soybean meal Brazil & 9.69 \\
& Soybean meal Argentina & 9.69 \\
& Other ingredients & 10.06 \\
Tototal & 100.00 \\
& Yellow corn & 38.71 \\
& Rice solvent bran & 19.59 \\
& Rice bran dry season & 12.89 \\
& Soybean meal Brazil & 8.34 \\
& Soybean meal Argentina & 7.46 \\
Other ingredients & 13.01 \\
Total & 100.00 \\
\hline
\end{tabular}

excluded during recipe calculation. The production cost of each recipe can vary due to different types of feedstuffs incorporated at different inclusion levels (Thomas et al., 1998). When different types of feedstuffs are processed, it results in different feed qualities and pelleting rates, hence, varying production costs. Operators need to adjust pelleting parameters such as die size and die pressure in order to accommodate the unique physical-chemical properties of the diet ingredients. The frequent change of recipes due to raw material price fluctuation causes a mill to frequently adjust their process parameters. However, correct adjustment of the process parameters is a difficult task because the interactions among each feedstuff and between the feedstuffs and production processes are very complex. As a result, a mill can only roughly estimate rather than 
exactly measure the effects of these parameters on pellet quality (Thomas et al., 1998) and slowly adjust their processes to accommodate changes in the physical-chemical properties of each feed ingredients by trial-and-error. The slow learning process often gives rise to feed production that does not meet mill's standards for pellet quality and often results in the mill having to reprocess the materials. Because the reprocess batches need extra clearing and extensive reprocessing time, dropping of the mill's capacity becomes one of the consequence of the high reprocess rate. Hence, highly fluctuated agricultural prices are often followed by high production costs for the mill. Moreover, the numbers of reprocessed batches also rise along with production completion time and back orders. The situation is likely to give rise to customer complaints and even a loss in sales if it persists (Pathumnakul et al., 2009).

Pathumnakul et al. (2009) suggested a way to prevent the reprocessing caused by such a situation by detecting the feed rations prior to starting actual production. They offer a prediction model created by the artificial neural network (ANN) as a tool for the production manager to communicate with the formulation manager such that the problematic feed rations can be distinguished from the good ones.

\section{Data collection}

For the ANN, Pathumnakul et al. (2009) recommended the use of a pelleting rate (ton/hr) and dust level (\%) as the output and the percentage of each main raw material (16 raw materials) included in feed formula and the percentage of molasses as input parameters of the model. They have tested the model with other input variables such as process parameters (pelleting mill number, die size, die thickness, feeder speed, press motor amperage, steam pressure and feed temperature), percent of nutrient contents in each main raw material type. As for industrial applications, using the percentage of main raw material and molasses as input parameters is the most practical model because some other factors such as the process parameters are not known during formula selection. It is more convenient for the nutrition manager to work on the model, which relies mainly on the least cost formulation output. Hence, the suggested set of parameters is best suited for the present business process.

The three-layer feed-forward back propagation neural network is used to conduct relationships between inputs and outputs. The commercial software MATLAB 6.5 was applied as a tool to develop the ANN model. The number of neurons in the input layer is equal to the number of input parameters, which are used to assess the production rate and the dust level. The number of neurons in the hidden layer is determined by investigating the number of neural networks ranging from 3 to 60 neurons.

\section{The production cost estimation process}

If the proposed cost estimation is to be implemented, the business process will be changed as shown in Figure 2 . Both approaches differ in the sense that, for the traditional approach, mills need to operate skeptical formulae in normal conditions even if they suspect that the pelleted products might not meet the mill's quality standards. These processes can be costly and time consuming. On the contrary, the proposed method requires using ANN to estimate the costs without an actual production run. After obtaining the leastcost ration, the nutrition manager can input all necessary information into the ANN and let it assesses the performance of the formulation instead of leaving this up to the individual operator. The least cost ration, which meets all pellet criteria will be obtained in a final step. It can potentially save time and costs for the mill.

Table 2 shows the use of ANN to obtain the production rate and dust level prediction in a feed mill in Thailand. The nutrition manager inputs the percent inclusion of the raw materials into the ANN-based software. Table 2a presents the input data of a swine-feed recipe. The software then converts these input parameters into an estimation of production rates and dust levels of two distinct pelleting lines (Table 2b). The two outputs, then, are converted to the costs of production, which is the summation of the estimated production costs and the raw material costs.

The estimated production cost is divided into total operating costs (i.e., direct labor and overhead cost) and reprocessing costs. The per-hour operating cost (baht/ pelleting machine-hour) is obtained from dividing the total operating cost by the total machine-hour. Then, the per-ton operating cost (Eq. (1)) is calculated by dividing the perhour operating cost by the estimated production rates (tons/hr) obtained from ANN. The reprocessing cost is incurred when the percent of dust level of the product exceeds its maximum allowance. Because the reprocessing incurs twice the operating cost, it is presumably equal to the per-ton operating cost. The mathematical expression of the per-ton total production cost is illustrated in equation (2).

$$
\begin{aligned}
& O C_{i}=\frac{A O C}{P R_{i}} \\
& P C_{i}=O C_{i}+O C_{i}\left(X_{i}\right)
\end{aligned}
$$

where $P C_{i}=$ the production cost of feed formula $i$ (baht/ t); $O C_{i}=$ the estimated operating cost of producing feed formula $i$ (baht/t); $P R_{i}=$ the production rate of feed formula $i(\mathrm{t} / \mathrm{hr}) ; A O C=$ the average per-hour operating cost (baht/ pelleting machine-hour) 

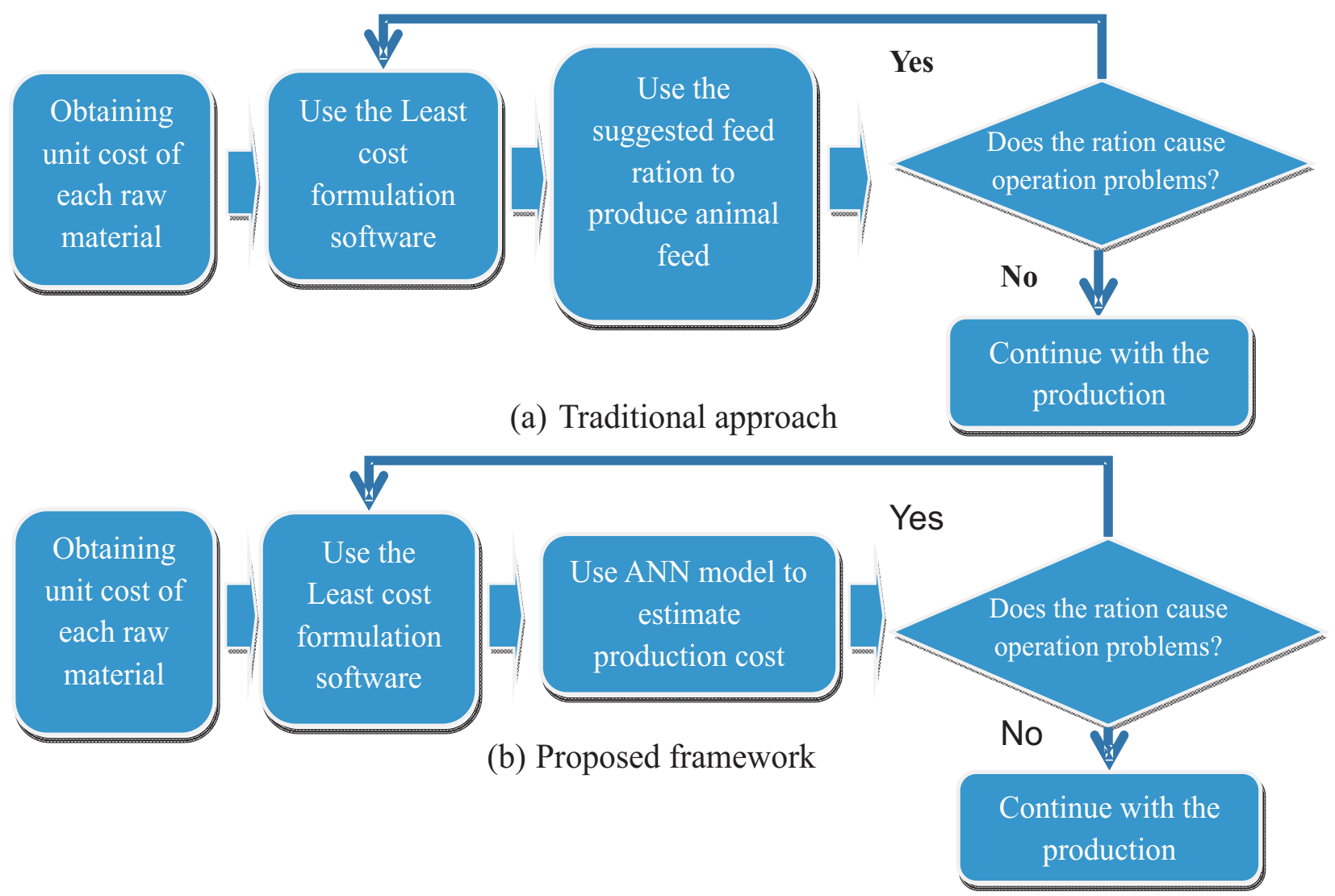

Pathumnakul et al. (2009)

Figure 2 - Framework for feed rations selection.

Table 2 - The use of ANN to estimate production rate and dust level

(a) The example of input data

Swine feed

Raw material \% inclusion

Yellow corn Thai 24.98

Soybean meal $\quad 20.04$

$\begin{array}{ll}\text { Dry season raw rice bran } & 18.00\end{array}$

Rice solvent bran $\quad 16.38$

$\begin{array}{ll}\text { Cassava chip } & 12.00\end{array}$

Molasses $\quad 2.00$

$\begin{array}{lr}\text { Crude palm oil } & 1.60\end{array}$

Others $\quad 5.00$

$\begin{array}{ll}\text { Total } & 100.00\end{array}$

(b) The example of results

Swine feed

Pelleting line Estimated production rate $(\mathrm{t} / \mathrm{h})$

Estimated dust level (\%)

Estimated production dost (baht/t)

\begin{tabular}{llcc}
\hline 1 & 16.44 & 4.01 & 386.41 \\
2 & 14.78 & 3.75 & 424.72 \\
Average & 15.61 & 3.88 & 405.6 \\
\hline
\end{tabular}




\section{$X_{i}= \begin{cases}1, & \text { if feed formular } i \text { required reprocessing } \\ 0, & \text { otherwise }\end{cases}$}

Implementing the new concept of production cost estimation

To implement the proposed method of production cost estimation, one needs to collate data from the production line and the nutrition department. Production rates and the quantity of each batch should be recorded along with the dust levels. Production rates can be obtained from subtracting the ending time from the starting time to retrieve total production time, then, divide the total production quantity. The dust level is measured by dividing dust quantity by the total of the sampled quantity. From our experience of working with feed mills, long-range data does not increase the accuracy of the prediction. That is because the mill might experience employee turnover or pellet equipment might be adjusted as a part of the technological improvements. Hence, some other parameters rather than the percent inclusion of raw material might affect the pelleting rate. The same recipe might end up with different pelleting rates. Hence, the long-range data incorporated into the dynamic change of feed mill environments might have a negative effect on prediction accuracy. From a practical standpoint, three months of the most recent data is a good range of data for the prediction of feed production costs. However, using short range of data might also cause another problem. Because the raw material varies by season, shortrange data (e.g., three months) is likely to leave out some of the raw material from the neural network. For example, cassava chip is used for certain months of the calendar year. Three months after the end of cassava harvest season would not be included in the neural network using the shortrange data. Hence, when the next season starts, the three month updated neural network will not recognize the effects of cassava on pelleting rate and quality. The three month updated neuron will treat it as a new raw material. To solve this problem, we constructed a database to collect one-year data. And if the 3-month data set used to train the neural network indicates unfamiliar raw materials, one can check if it is really new or if it is only excluded from the data set because of seasonality. If the mill used that raw material in the past, its performance can be also included in the train data set. Hence, the train data set is now composed of 3 months of the current data plus the formulae that produced in the past but excluded from this 3-month data set due to its seasonality. Moreover, not all data is appropriate to be incorporated in the neural network. Some data includes outliers due to human error or some other factors. Bad data can create the problem of garbage-in-garbage-out causing prediction inaccuracy. Therefore, the outliers should be detected prior to the learning process of the neural network.

Because implementing the proposed cost estimation involves parties such as the nutrition and operation departments or perhaps sale department, the concept the estimation should be clarified among all parties. Meetings and guidelines should be conducted and agreed on at the beginning of the project to enhance the success of the project. Executive involvement will urge all parties to realize the need for a new cost estimation project. Capability to estimate production costs will facilitate the mill in determining the pricing strategies. Hence, it should also enhance competitiveness of the mill.

After using the ANN for couple of months, prediction accuracy should again be investigated and another meeting should be constructed to improve the model.

The use of production rate estimation in feed scheduling

Being able to estimate the production rate can also be used to facilitate production scheduling and its use is described next.

\section{The needs for accurate estimation of production rate}

Due to highly competitive environment, the feed mills face with an increasing number of feed formulations and variety of feed products which tailor-made toward clientspecific needs and requirements. As a result of the feed industry responds to market trends, their production processes become more complex. To cope with these complexities, the feed mills need to be more flexible and more efficient in coordinating their resources. As the operations are sequence-dependent, product sequencing is critical for being efficient. This feed scheduling problem is similar to the sequencing restrictions found in the food industry. The mill produces numerous products that differ in package size, nutrient contents, or presence of ingredients that increase the functional value. The products can be grouped according to their transition cost and time into; non-drug and drug; pelleted, powder and crumble; high fiber and low fiber; and bulk and packed. The production time is not uniform among the products. The factors contribute to nonuniformity production speed, especially in pelleting machine, is the ingredients of the products. For example, fat contents enhance lubrication of the products and, hence, the speed. The fiber contents have the characteristic of being stiffness and elastics which might inhibit good contact between particles in the feed (Thomas et al., 1998). Hence, fibrous products may lead to lower production speed. Some product families contaminate other if produced in successive batches. As a result of increasing wide variety of the product, the production line must be often cleaned, 
consuming potential production time. Usually, products in the same family have negligible changeover times, and identical batch weights and processing times. This amount of mixer cleaning time can be minimized by good sequencing of the production of families (Taso et al., 2009). The example of products that might create different changeover times for different sequencing is a formula with/without drugs. Generally, a formula with drugs should be processed before a formula with drugs. The opposite would require an extra flushing operation to ensure that no drugs would be transferred to the non-drug formulae. In this light, changes in the packaging materials; and configuration and cleaning the machinery during transition of feed formula require a changeover time and cost. Hence, an optimal schedule would reduce the cost and time incurring during changing over.

\section{Feed processes}

To understand the role of production rate estimation in scheduling, one needs to understand the processing first. Generally, the feed production process is divided into three steps: mixing, pelleting, and packing (Figure 3).

In the mixing stage, bulk ingredients such as soybean meal and some other micro ingredients such as vitamins or medications stored in waiting bins are weighed according to feed ratio. The feed ingredients which are coarse cereal grains such as corn required a grinding process, so, it is delivered to hammer mills. Grinding will reduce grain size and expose surface area of the particles allowing digestive enzymes to access nutritional components such as starch and protein resulting in better absorption in the digestive tract of the animal and increase animal performance as well. Moreover, as a result of size reduction, the modified physical characteristics of ingredients will improve mixing, pelleting, handling, and transportation process. After grinding process, dry meal particles are blending with liquids ingredients (such as added fat) or others ingredients in a mixer.

Next, the feed mash is conveyed to pelleting mills. In the pelleting process, a soft feed mash is forced through holes in a metal die plate to form compacted pellets, and then, cut to a pre-determined size. Pelleting is a crucial process in the feed production. The high quality pelleted products ensure the correct amount of nutrition for all that consume them each and every bite. The thickness of the die plate helps to determine the compactness and stability of the pellet. In this process, an operator needs to change die size according to the mill's standard. Therefore, choosing die size is product-dependent and should be accommodated in the

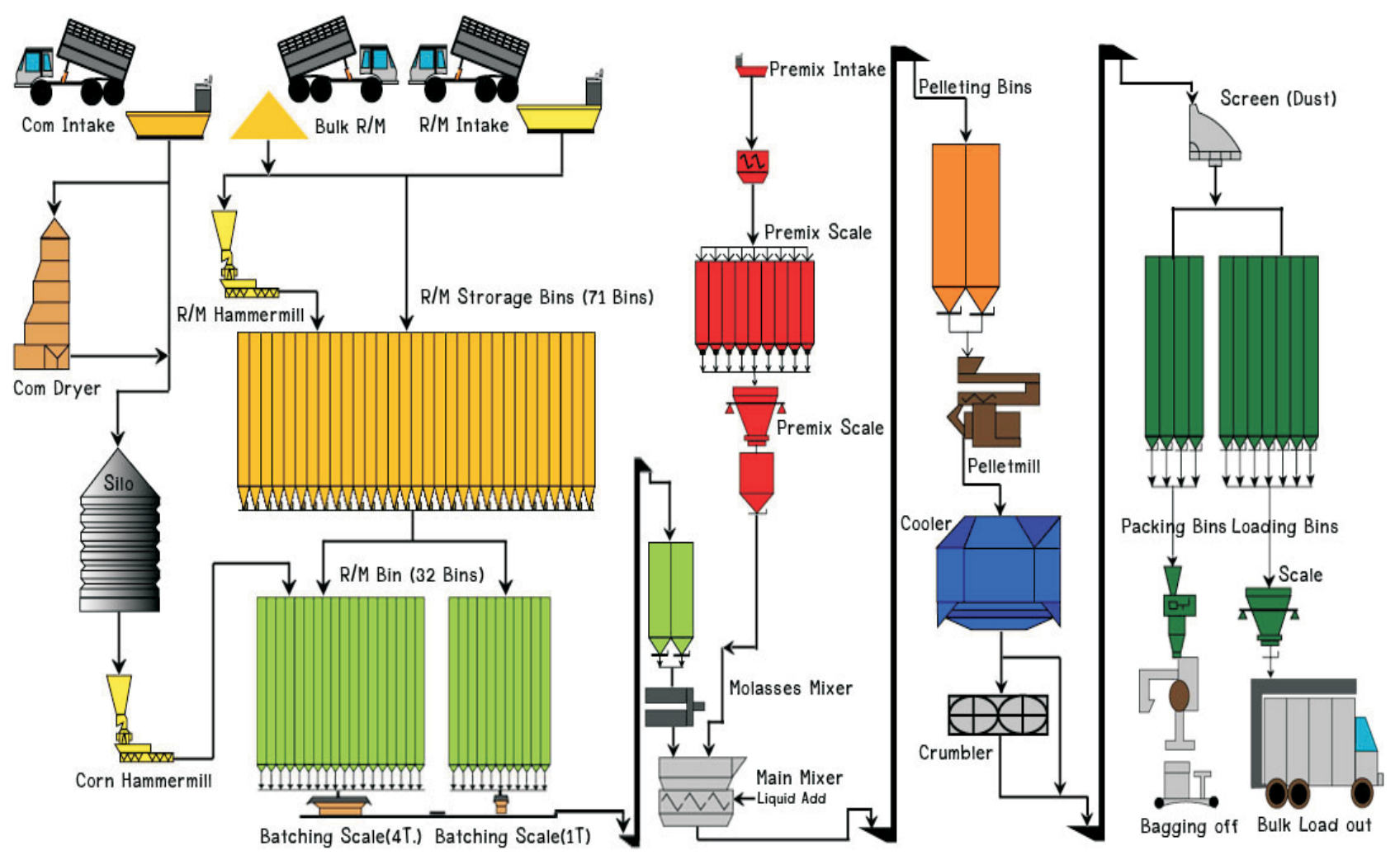

Figure 3 - Feed processing system. 
scheduling. Changeover time of die size is sequence dependent. If all products are placed in a succession where the change of die size is minimized, the capacity of the machine will, in turn, increase. From there, the pelleted products are transferred to a cooler/drier to remove the heat which is generated during the pelleting process and the steam conditioning in a mixing process. The moisture content of the pellets also needs to be reduced to $10-12 \%$ or less for proper storage and handling. Pellets must therefore be cooled and dried. From the finished product bins the finished feed is either be bulk transport, loading directly onto trucks or it will be packed into bags ( 30 and $50 \mathrm{~kg}$.). The smaller bag takes longer time to pack, hence, should be also considered in the scheduling goal.

In the case that a feed mill operates with one mixer, several pelleting machines and packings, a planner needs to consider the following factors: The feed production being scheduled should not exceed capacity of each machine; The drug free formulae should be processed before a formula with drugs; The production should meet demand requirements; The pelleting machine should be sequenced to minimize the change over time; While the packing machine is packed, bulk production can be processed such that ideal time of pelleting machine is minimized; Mash production does not require pelleting. Hence, its production can be on parallel with pelleted products. From this complication, one can notice that ability to estimate is crucial for effective scheduling. Inappropriate of production rate estimation could lead to scheduling solutions with high cost or inappropriate timing and infeasible schedules respectively. A planner needs to decide the sequence of the formulae into the mixer and from the mixer what pelleting machine should be utilized. The appropriate timing then relies on the estimation of pelleting rate. The inaccurate estimation of the rate might lead to under utilizing of machine time, hence, lost of opportunity cost to the mill.
The benefits of prediction accuracy: an illustrative case

Traditionally, the production rate used in feed scheduling is obtained by planner's experience. The obtained rate is approximately the average of rate of production produced in the past. This practice leads to unintentional lost in production cost and time, if the actual production rate of each feed recipe differs from the average production rate. In an example of 2 pelleting lines called PM1 and PM2, the average production rate for PM1 is 12 tons/hr and it is 17 tons/hr for PM2. Assume that feed mill was to produce 10 batches of 10 distinct feed products. The quantity production of each batch is approximately 50 tons. The production rate of each product is different from one another due to its raw material composition (Table 3 ). The clearing time required in changing one recipe to another is 30 minutes. The optimal product sequencing of the two pelleting machines is contructed using the average production rate will be different from the optimal sequencing formulated based on a more accurate estimation of production rate. In this sample case, the total processing time and system completion time of feed scheduling based on average capacity are 2,520 and 1,552 minutes, respectively, while they are 2,274 and 1,261 minutes, for sequencing based on a more accurate production rate. The use of inaccurate production rate leads to longer total processing time and completion time at approximately $9.76 \%$ and $18.75 \%$, respectively (Figure 4a, b). The longer times of using inaccurate production rate in scheduling imply the potential cost saving from using a more accurate estimation of production rate. The cost saving is even more shining in the case of agricultural price turbulent. That is because the frequent change of recipe composition will make it harder for planner to anticipate the change of production rate. Hence, inappropriate scheduling is likely to result from the unanticipated rate.

Table 3 - Actual production rates in example case

\begin{tabular}{|c|c|c|c|c|c|}
\hline \multirow[t]{2}{*}{ Feed products } & \multicolumn{2}{|c|}{ Actual production rate $(\mathrm{t} / \mathrm{h})$} & \multirow[t]{2}{*}{ Feed products } & \multicolumn{2}{|c|}{ Actual production rate $(\mathrm{t} / \mathrm{h})$} \\
\hline & PM 1 & PM 2 & & PM 1 & P M 2 \\
\hline 1 & 10.50 & 19.00 & 6 & 8.80 & 16.00 \\
\hline 2 & 8.50 & 15.50 & 7 & 10.00 & 18.80 \\
\hline 3 & 14.50 & 20.20 & 8 & 11.50 & 18.00 \\
\hline 4 & 12.70 & 18.40 & 9 & 8.80 & 15.40 \\
\hline 5 & 14.20 & 19.50 & 10 & 13.30 & 19.00 \\
\hline
\end{tabular}




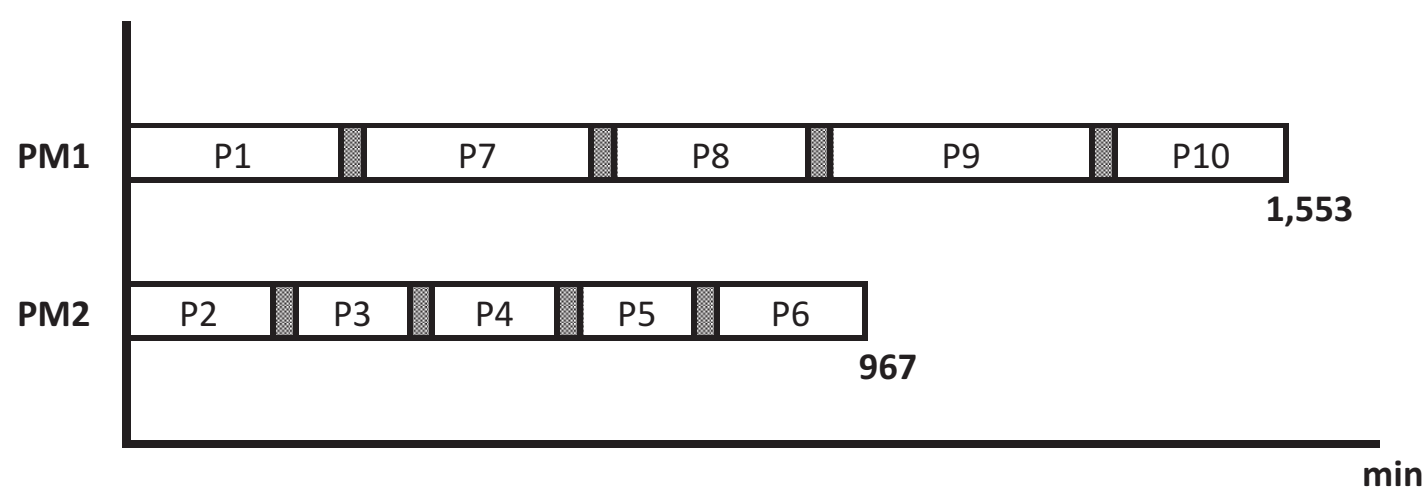

(a) The product sequence based on the average production rate

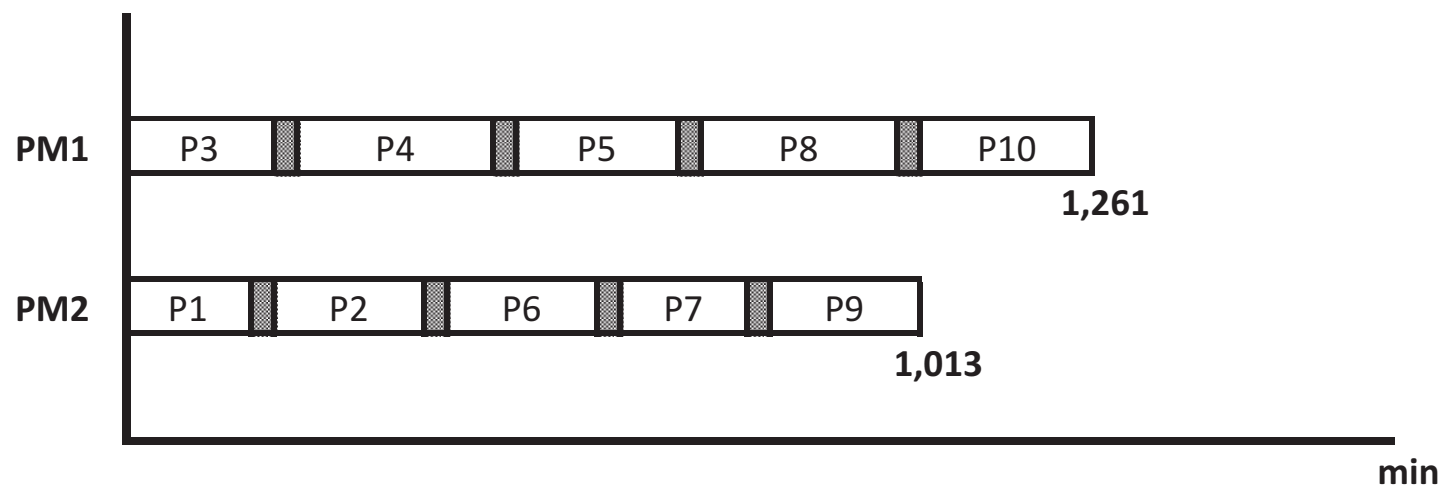

(b) The product sequence based on actual production rate

Figure 4 - The comparison between product sequences based on average and actual production rates.

\section{Conclusions}

In this study, we demonstrate the effects of agricultural price fluctuation on feed cost estimation as well as production scheduling. It is found that the agricultural price fluctuation lead to frequent change of feed recipe. And the combination of inappropriate process adjustment and frequent change of feed recipe causes high number of reprocessed batches, and hence, per unit production cost. To deal with this problem, we offer an estimation method based on artificial neural network to predict production rate and cost prior an actual production. The estimated production rate is not only useful for cost estimation but also feed scheduling. Capability to estimate production facilitates good sequencing of feed production. Change over time and cost can be reduced as a result. Although cost estimation and feed scheduling are the only two applications of feed production estimation, it can also be applied to other aspects of feed production (i.e., total productivity improvement, process adjustment) as well. The other applications have yet but worth been explored.

\section{Acknowledgments}

This research was funded by The Thailand Research Fund under project IUG51 and Betagro Science Center Company Limited. The authors also acknowledge the company's help in collecting data, providing invaluable guidance and making this research possible.

\section{References}

PATHUMNAKUL, S.; PIEWTHONGNGAM, K.; APICHOTTANAKUL, A. A neural network approach to the selection of feed mix in the feed industry. Computers and Electronics in Agriculture, v.68, n.1, p.18-24, 2009.

THOMAS, M.; VLIET, T.; VAN, POEL, A.F.B. Physical quality of pelleted animal feed. 3. Contribution of feedstuff components. Animal Feed Science Technology, v.70, p.59-78, 1998.

TOSO, E.A.V.; MORABITO, R.; CLARK, A.R. Lot sizing and sequencing optimization at an animal-feed plant. Computers and Industrial Engineering, v.57, p.813-821, 2009. 